\title{
Laparoscopic Transperitoneoscopic Nephroureterectomy in a Patient with Situs Inversus Totalis
}

\author{
Kaan Gökçen • Hüseyin Çelik • Murat Kobaner • \\ Sinan Karazindiyanoğlu
}

Received: 26 June 2014 / Accepted: 5 January 2015 / Published online: 20 January 2015

(C) Association of Surgeons of India 2015

\begin{abstract}
This article discusses the case of a 78-year-old female with painless gross hematuria. Chest X-rays showed dextrocardia with situs inversus, and whole-body computed tomography scanning showed a renal mass in the right renal pelvis, no evidence of metastasis, and mirror-image organs with left-to-right transposition, which resulted in a diagnosis of situs inversus totalis (SIT). A laparoscopic transperitoneoscopic right nephroureterectomy was scheduled. To our knowledge, our case is the second case of laparoscopic nephroureterectomy in renal pelvic urothelial carcinoma with SIT to be presented, but it is the first case of laparoscopic transperitoneoscopic nephroureterectomy. Laparoscopic transperitoneoscopic nephroureterectomy was successfully performed in a renal pelvic urothelial carcinoma patient with SIT with a correct description of renal vascularity and abdominal anatomy.
\end{abstract}

Keywords Laparoscopy $\cdot$ Nephroureterectomy $\cdot$ Situs inversus totalis · Urotelial cancer

\section{Introduction}

Situs inversus totalis (SIT) is a rare condition in which there is a complete transposition (right to left reversal) of the thoracic and abdominal organs. It is a congenital anomaly and generally an autosomal recessive genetic condition. Its incidence is

K. Gökçen · M. Kobaner · S. Karazindiyanoğlu

Department of Urology, Osmaniye State Hospital, Osmaniye, Turkey

H. Çelik $(\triangle)$

Department of Urology, Inonu University Turgut Ozal Medical

Center, Malatya, Turkey

e-mail: drhuseyin@hotmail.com approximately one in every 10,000 to 50,000 live births. SIT has no pathophysiological significance, and the survival rate of patients with SIT does not differ from that of healthy persons $[1,2]$.

Situs inversus totalis may pose several technical difficulties during operative procedures, especially during laparoscopic operations. The mirror image of the laparoscopic view creates an unfamiliarity for surgeons and their usual maneuvers. In addition, all of the instrument designs are for right-handed surgeons [3]. We present a case of a transperitoneoscopic nephroureterectomy involving a renal pelvis urothelial carcinoma patient with SIT.

\section{Case Report}

A 78-year-old female presented to our clinic with painless gross hematuria. During the preoperative investigation, radiologic imaging studies showed the presence of complete SIT. The results of the blood biochemistry and urine tests were in the normal ranges. Chest X-rays showed dextrocardia with situs inversus, and whole-body computed tomography (CT) scanning showed a renal mass in the right renal pelvis, no evidence of metastasis, and mirror-image organs with left-toright transposition, which resulted in a diagnosis of SIT (Fig. 1a-b). The urine cytology was positive for urotelial cancer. The ureteroscopic biopsy of the right renal pelvic mass confirmed a high-grade papillary urotelial cancer. The patient's preoperative staging was cT1N0M0. The CT angiography with three-dimensional reconstruction demonstrated a prior branching in the right renal artery (Fig. 1c-d).

A laparoscopic transperitoneal right nephroureterectomy was scheduled. Pneumoperitoneum was achieved using a Veress needle. We placed three $12-\mathrm{mm}$ trocars and one 5$\mathrm{mm}$ trocar. After the white line of the Toldt line incision, the colon was mobilized medially up to the splenic flexure on the 
Fig. 1 a-b Computed tomography scan demonstrated a mass in the right renal pelvis. $\mathbf{c}-\mathbf{d}$ 3D Reconstruction of an abdominal computed tomography angiogram showing the kidney's arterial structure. c Anterior side. d Posterior side
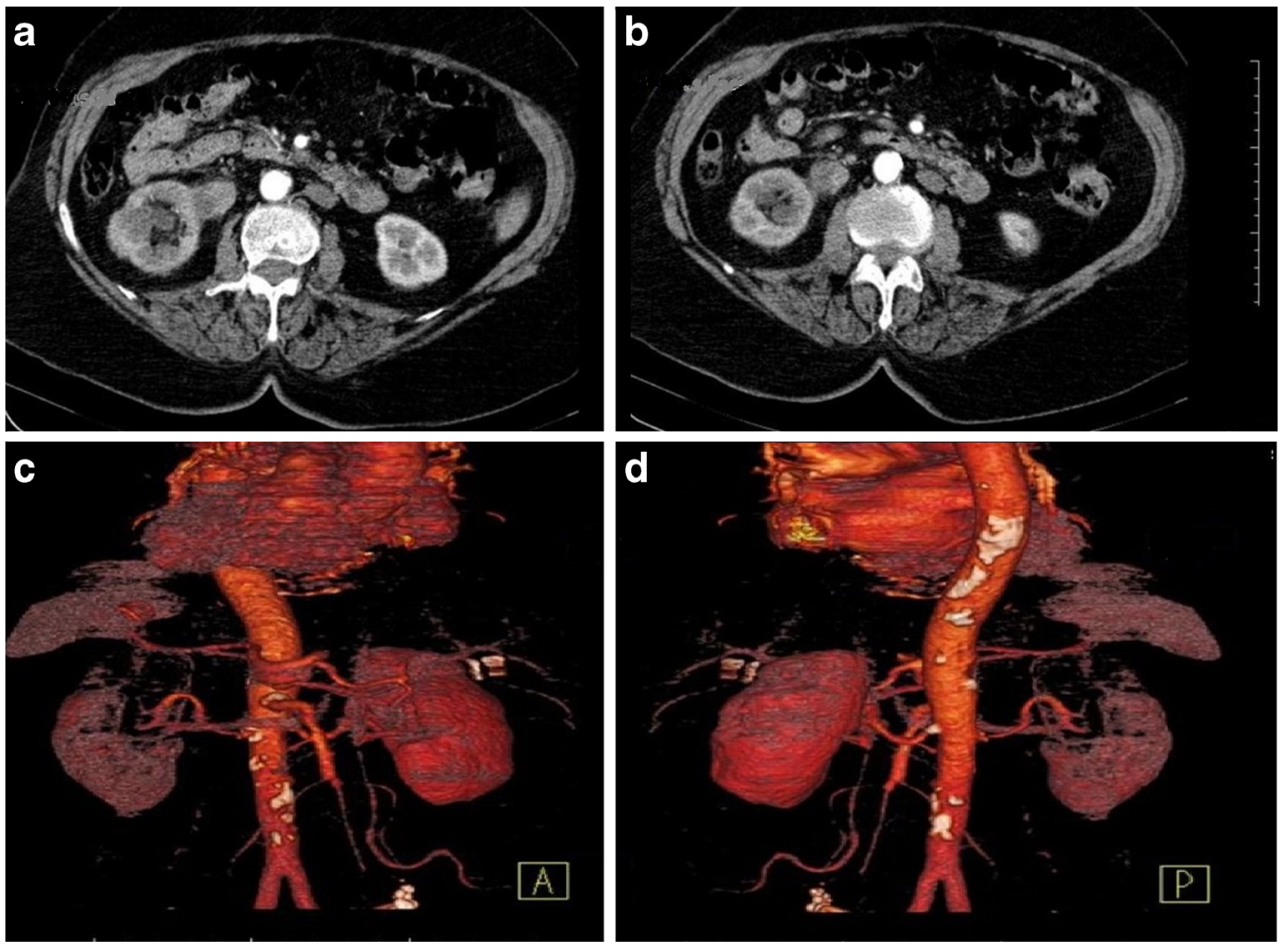

right side. The right renal vein, the artery, and the other venous vessels (including the gonadal, the right adrenal, and the lumbar veins) were anatomically consistent with those of the left side. After the hilar dissection and the exposure of the renal artery and the vein, the kidney was released from the surrounding structures and the adrenal gland. Following the right renal pedicule dissection, the kidney was released from the structures (Fig. 2a). The ureter was dissected down to the bladder. A lymphadenectomy of the right side was performed. After patient position was changed to the supine, we performed a bladder cuff excision and removed the specimen through the small Gibson incision (Fig. 2b).

The insufflation time was $110 \mathrm{~min}$, and the estimated blood loss during the surgery was $50 \mathrm{~mL}$. There were no intraoperative or postoperative complications. The Foley drain was removed on the third postoperative day, and the patient was discharged from the hospital on the fifth postoperative day. A pathologic examination revealed a high-grade, invasive urothelial carcinoma of the right renal pelvis with a squamous differentiation in the focal area. Six lymph nodes were extracted from the specimen, and none of the lymph nodes were involved in the cancer. The pathological staging was pT1N0M0. The patient came for control at intervals of 3 and 6 months. The patient had no complaints.

\section{Discussion}

Situs inversus totalis (SIT) is a rare congenital malformation characterized by a transposition of organs to the opposite side of the body in a mirror image of the usual $[1,2]$. Many laparoscopic procedures have been reported to be performed safely in patients with SIT despite

Fig. 2 a Renal hilum and spleen in the right side of the abdomen. $\mathbf{b}$ Trocar sites and Gibson incision
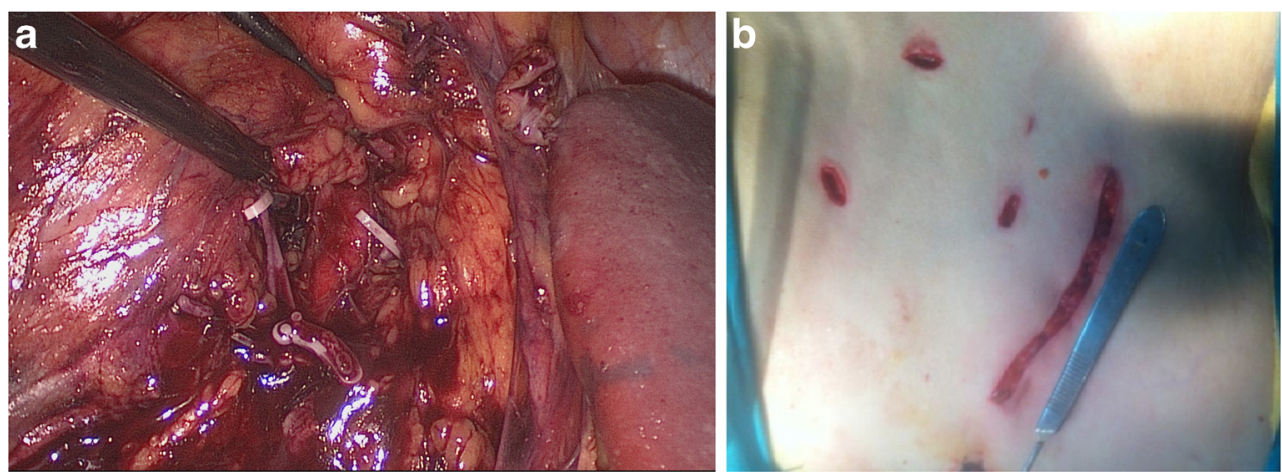
the fact that there are some technical difficulties due to the mirror image of the anatomy [3].

In the urology, both a complete laparoscopic nephrectomy and a partial nephrectomy in a patient with SIT have been reported [4]. There are some articles about laparoscopic donor nephrectomies with SIT $[5,6]$. The articles reported that SIT is not a barrier in performing these surgeries. In order to perform a successful surgery, it is necessary to describe the patient anatomy before the operation. Some preoperation imaging techniques, such as $\mathrm{CT}$ and $\mathrm{CT}$ angiography, are useful for surgeries. Makiyama et al. reported the first case of laparoscopic retroperitoneoscopic nephroureterectomy in renal pelvic urothelial carcinoma in patients with SIT. They used preoperative training using a patient-specific simulator [7]. Our case is the second case of laparoscopic nephroureterectomy reported in a patient with SIT, but it is the first case of laparoscopic transperitoneoscopic nephroureterectomy reported. We used CT and CT angiography prior to the operation and almost memorized the patient's abdominal anatomy. We considered this case to be an anatomic variant, and the operation was performed. We were able to confirm that the anatomy of the patient was almost identically reproduced in the CT and CT angiography in comparison with the intraoperative findings. We were able to complete the right transperitoneoscopic nephroureterectomy without any complications. The operation time was rational.

\section{Conclusion}

We have shown that laparoscopic transperitoneoscopic nephroureterectomy can be performed safely in patients with SIT. Also, SIT is not an impediment in performing these procedures. We suggest that patients with SIT be evaluated preoperatively with detailed imaging studies prior to the laparoscopic nephroureterectomy process. As a result, laparoscopic transperitoneoscopic nephroureterectomy would be feasible with the correct description of the renal vascularity and the abdominal anatomy.

Acknowledgments There is no financial support.

Conflict of Interest The authors declare no conflict of interest related to this article.

\section{References}

1. Douard R, Feldman A, Bargy F, Loric S, Delmas V (2000) Anomalies of lateralization in man: a case of total situs inversus. Surg Radiol Anat 22:293-297

2. Fernandes MN, Neiva IN, de Assis CF, Meguins LC, Fernandes MN, Meguins EM (2008) Three-port laparoscopic cholecystectomy in a Brazilian Amazon woman with situs inversus totalis: surgical approach. Case Rep Gastroenterol 2(2):170-174

3. Fujiwara Y, Fukunaga Y, Higashino M, Tanimura S, Takemura M, Tanaka Y, Osugi H (2007) Laparoscopic hemicolectomy in a patient with situs inversus totalis. World J Gastroenterol 13:5035-5037

4. Wang LH, Liu B, Wu Z, Yang Q, Xiao L, Chen W, Sheng H, Luo W, Luo R, Sun Y (2011) Left transperitoneal laparoscopic partial nephrectomy in the presence of a left-sided inferior vena cava. Urology 78 : 469-473

5. Berber I, Gures N, Gurluler E, Alim A, Cakir U, Gurkan A (2013) Total laparoscopic donor nephrectomy in situs inversus totalis: a case report. Exp Clin Transplant 11(2):195-198

6. Simforoosh N, Beigi FM, Aminsharifi A, Shayaninasab H, Mehrabi S (2007) Left-sided inferior vena cava found incidentally during laparoscopic donor nephrectomy: report of three cases. J Endourol 21:542544

7. Makiyama K, Sakata R, Yamanaka H, Tatenuma T, Sano F, Kubota Y (2012) Laparoscopic nephroureterectomy in renal pelvic urothelial carcinoma with situs inversus totalis: preoperative training using a patient-specific simulator. Urology 80(6):1375-1378 